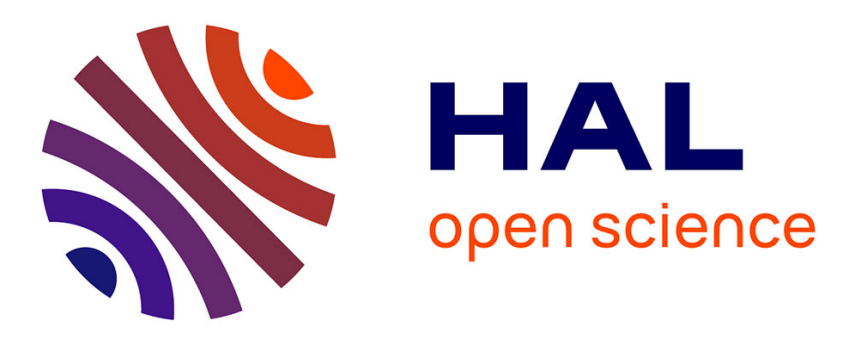

\title{
Bubble Production Mechanism in a Microfluidic Foam Generator
}

Maximilien Stoffel, Sebastian Wahl, Elise Lorenceau, Reinhard Höhler, Bruno Mercier, Dan E. Angelescu

\section{- To cite this version:}

Maximilien Stoffel, Sebastian Wahl, Elise Lorenceau, Reinhard Höhler, Bruno Mercier, et al.. Bubble Production Mechanism in a Microfluidic Foam Generator. Physical Review Letters, 2012, 108, pp.198302. 10.1103/PhysRevLett.108.198302 . hal-00785723

\section{HAL Id: hal-00785723 \\ https://hal.science/hal-00785723}

Submitted on 11 Apr 2013

HAL is a multi-disciplinary open access archive for the deposit and dissemination of scientific research documents, whether they are published or not. The documents may come from teaching and research institutions in France or abroad, or from public or private research centers.
L'archive ouverte pluridisciplinaire HAL, est destinée au dépôt et à la diffusion de documents scientifiques de niveau recherche, publiés ou non, émanant des établissements d'enseignement et de recherche français ou étrangers, des laboratoires publics ou privés. 


\title{
Bubble production mechanism in a microfluidic foam generator
}

\author{
M. Stoffel,${ }^{1}$ S. Wahl,${ }^{1}$ E. Lorenceau, ${ }^{2}$ R. Höhler,${ }^{3,2}$ B. Mercier, ${ }^{1}$ and D.E. Angelescu ${ }^{* 1}$ \\ ${ }^{1}$ Université Paris-Est, ESIEE Paris/ESYCOM, Noisy le Grand, 93162 France \\ ${ }^{2}$ Université Paris-Est, LPMDI FRE 3300 CNRS, Champs-sur-Marne, 77420 France \\ ${ }^{3}$ Université Paris 6, INSP, UMR 7588 CNRS-UPMC, Paris 75252, France
}

(Dated: March 21, 2012)

\begin{abstract}
We present the design and characterization of a microfluidic bubble generator that has the potential of producing monodisperse bubbles in 256 production channels that can operate in parallel. For a single production channel we demonstrate a production rate of up to $4 \mathrm{kHz}$ with a coefficient of variation of less than $1 \%$. We observe a two-stage bubble production mechanism: initially the gas spreads onto a shallow terrace, and then overflows into a larger foam collection channel; pinning of the liquid-gas meniscus is observed at the terrace edge, the result being an asymmetric pinch-off. A semi-empirical physical model predicts the scaling of bubble size with fluid viscosity and gas pressure from measurements of the pinned meniscus width.
\end{abstract}

The generation of dispersions using microfluidic devices has seen a surge of interest in recent years due to the excellent control that can be obtained over the droplet size and monodispersity of the dispersed phase. Different types of confined two-dimensional device geometries have been attempted, T-junctions $[1,2]$ and flow focusing approaches [3-7] being the most common. Such devices can be used to generate uniform dispersions (coefficient of variation $C V \approx 2-5 \%$ ), with sizes of order $10 \mu \mathrm{m}$ and production frequencies of a few $\mathrm{kHz}$ for $\mathrm{T}$-junction systems [1] and upwards of $100 \mathrm{kHz}$ for flow-focusing geometries $[6,7]$, but they do not allow individual control of these two parameters due to their dependence on imposed flowrates. Attempts to achieve higher throughput (up to the $\mathrm{MHz}$ range) have been made by parallelization in both types of geometries [8,9] , but cross-talk between neighboring production sites $[10,11]$ limit the control over the size of resulting objects. Such effects are amplified when gas is used as the dispersed phase, due to the high compressibility of the gas which leads to strong hydrodynamic feedback even in the case of a unique production site [12].

Recently, more complex device geometries have been reported, which evade from two-dimensional limitations by combining confined and unconfined regions. Such "2.5D" geometries have proven very effective in generating controlled dispersions at high production rates [1316], with independent control of dispersion size (mostly imposed by geometric details) and production frequency (controlled by dispersed phase flowrate). Several studies have been made in liquid-liquid systems to explain the droplet production mechanism in such " $2.5 \mathrm{D}$ " geometries (also called microchannel emulsification devices) [17-19]. Both experiments and modeling results identify the viscosity ratio $\xi=\eta_{d} / \eta_{c}$ between the dispersed and the continuous phases as an important parameter governing

\footnotetext{
${ }^{*}$ Corresponding author, E-mail: d.angelescu@esiee.fr
}

device behavior [19]. In particular, they predict the existence of a geometry-dependent viscosity ratio threshold $\xi_{\text {min }}$ below which droplet formation becomes impossible; the lowest reported value of this threshold in liquid-liquid systems is 0.16 [19], suggesting that theoretically such geometries cannot be used to generate gas dispersions or foams.

In this Letter we report on the capability of a fully integrated microchannel emulsification device to generate highly monodisperse foams, at viscosity ratios significantly lower than previously reported threshold values. While our device contained 256 parallel production channels (thus being capable of high throughput), the focus of this study was on the physical characterization of bubble production at an individual channel. We investigated the dependance of the production frequency, droplet size, and $C V$, on process parameters such as gas pressure, liquid flowrate, and liquid viscosity. We demonstrate that high production frequencies $(4 \mathrm{kHz}$ per channel) of $\mathrm{pL}$ bubbles can be achieved with excellent monodispersity $(C V \approx 1 \%)$ and little dependence on flowrate or pressure. High-throughput operation with all channels producing bubbles in parallel results in higher polydispersity due to rapid foam aging and isolated coalescence in the outlet channel, phenomena currently under investigation. Interestingly, we show that the behavior of the contact line plays an unexpected role in the process of bubble formation, pinning at sharp angles on the device geometry being largely responsible for the observed phenomenology. We propose a semi-empirical model which captures, at least qualitatively, the observed behavior.

We used a microchannel emulsification device geometry inspired by the work of Sugiura [14]. Fabrication was performed in silicon using multiple-depth deep reactive ion etching to create the channel and terrace structures as well as the fluid access holes; two different geometries were fabricated and used in this study, having different terrace etch depths $\left(h_{1}=2 \mu \mathrm{m}\right.$ and $\left.h_{2}=2.5 \mu \mathrm{m}\right)$ but equal terrace widths $\left(w_{t}=11.6 \mu \mathrm{m}\right)$. The other geometrical parameters are summarized schematically in Figure 


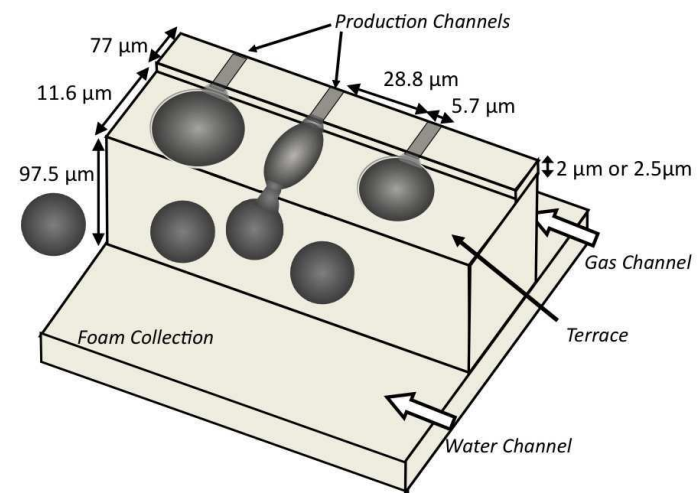

FIG. 1: Schematic of the device geometry (not to scale), showing three consecutive production channels. The glass wafer which seals the device at the top was not represented.

1. Device sealing was achieved by anodic bonding using a glass wafer, the resulting device being fully integrated onchip. A third device geometry was fabricated to test the predictions of our model, for which the terrace width increased gradually $(8 \mu \mathrm{m}$ at one end and $54 \mu \mathrm{m}$ at the other end of the device); the terrace etch depth was $2.5 \mu \mathrm{m}$.

As the dispersed phase we used nitrogen gas, the continuous phases consisting of solutions of deionized water, surfactant (sodium dodecyl sulfate $1 \% \mathrm{w} / \mathrm{w}$ ) and glycerol $(0 \%, 30 \%$ and $60 \% \mathrm{w} / \mathrm{w}$, corresponding viscosities: $0.95,2.35$ and $10.1 \mathrm{mPa} \cdot \mathrm{s})$. The corresponding viscosity ratios $\xi_{0 \%}=0.0189, \xi_{30 \%}=0.0077$ and $\xi_{60 \%}=0.0018$ were far lower than previously reported values of $\xi_{\min }$ in liquid-liquid systems; nevertheless our liquid-gas device was capable to reliably produce monodisperse bubbles.

The bubble formation process, shown at the top of Figure 2, displays several phases: initially, the circular gas pancake is fully contained within the device terrace (0ms $<t<50 \mathrm{~ms})$. As the radius of the pancake $R$ increases, the contact line touches the terrace edge and becomes pinned to it at $t=50 \mathrm{~ms}$, corresponding to $R=R_{\text {Touch }}$. After this point, the pancake extends laterally along the terrace until the final pinch-off of the bubble $(t=135 \mathrm{~ms})$. The in-plane radius of curvature (as indicated by dashed circles) constantly decreases during this time, and reaches a minimum $R_{\min }$ value just before pinch-off. The pinch-off is very rapid (less than $50 \mu \mathrm{s}$ for the $0 \%$ solution, up to $200 \mu$ s for the $60 \%$ solution), after which the pancake retracts $(t=136 \mathrm{~ms})$ and the process repeats cyclically. The variation of the pancake lateral radius of curvature is represented in Figure $2 \mathrm{a}$; we notice there that the minimal radius of curvature $R_{\text {min }}$ before pinch-off is independent of the glycerol concentration, and hence of viscosity. The production frequencies, however, do depend on both viscosity and gas pressure - the highest frequency recorded in our device was $4.1 \mathrm{kHz}$, suggesting a $\mathrm{MHz}$ maximum theoretical throughput for the 256 channels operating in paral-

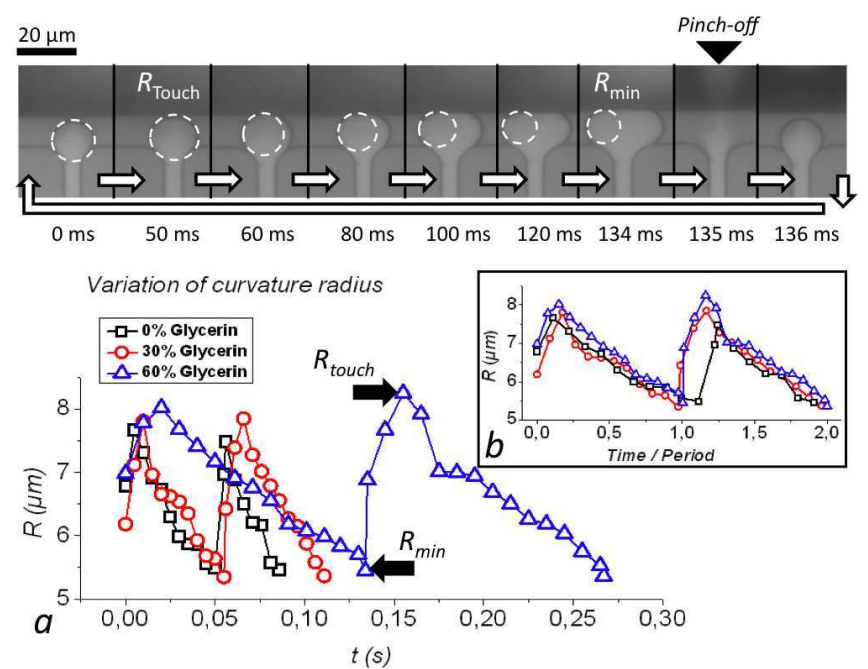

FIG. 2: Top: The production cycle of a bubble (using the $2.5 \mu \mathrm{m}$ device, a solution of $60 \%$ glycerol, gas pressure $P=$ $0.5 \mathrm{bar}$, and liquid flowrate $\phi=50 \mu \mathrm{l} / \mathrm{min}$ ). The white dashed circles are guides to the eye. Bottom: The variation of the curvature radius $R$ as a function of real time (a), and of time scaled using the production period (b), for the three glycerol solutions used.

lel. In Figure 2b, time was normalized by the production period corresponding to each viscosity. The overlap of the curves suggests that viscosity slows down the dynamics without otherwise affecting the bubble generation process. $R_{\min }$ was also found to be largely independent of the gas gauge pressure $P$, and of the fluid flowrate $\phi$ (the variation was less than $10 \%$ in the range 0.4 bar $<P<1.1$ bar, $10 \mu \mathrm{l} / \mathrm{min}<\phi<100 \mu \mathrm{l} / \mathrm{min})$. It is apparent from these cumulated observations that the "pancake spreading" stage of the bubble formation process is controlled primarily by device geometry and not by process parameters.

The pinning of the gas-liquid contact line to the edge of the terrace is critical in understanding the "pancake spreading" phase of the production process. As indicated schematically in Figure 3a, prior to pinning, the watergas meniscus advances on the terrace relatively slowly (dashed line on Fig. 3a), the contact with the glass and silicon being made at the respective advancing contact angles, $\theta_{g}$ and $\theta_{s}$. Once the interface becomes pinned to the terrace edge, the apparent silicon contact angle becomes free to take any value between the advancing contact angle for the horizontal and for the vertical silicon surface. As gas pressure increases, the pinned meniscus continues to advance slightly along the glass surface (keeping the same contact angle $\theta_{g}$ ), while the apparent contact angle at the silicon surface decreases. This slight movement initially leads to an increase in out-ofplane curvature, the minimum radius of curvature being reached when the meniscus becomes horizontal at the 

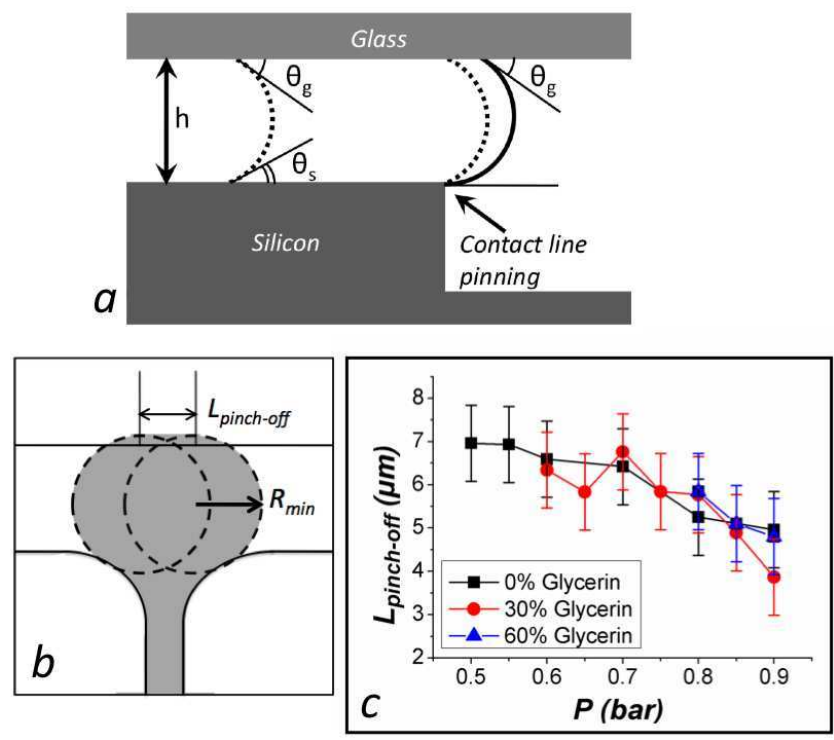

FIG. 3: Schematic of the pinning process at the terrace edge (lateral view: a, top view: b). The length of the pinned meniscus just before pinch-off $\left(L_{\text {pinch-off }}\right)$ as a function of gas pressure for the three solutions used.
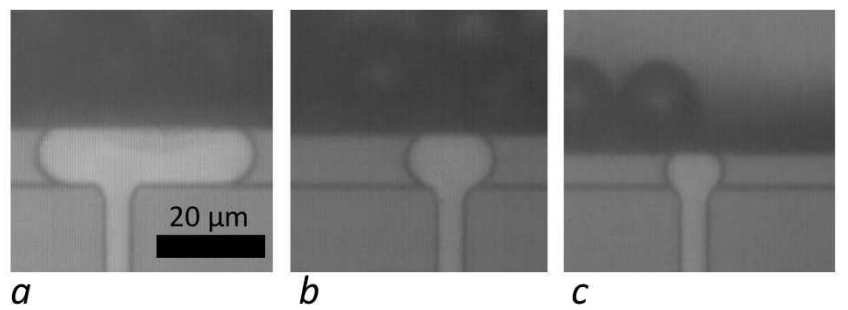

FIG. 4: The dependance of the "pancake spreading" process on terrace width, observed in the variable geometry device here three different widths are shown (a: $14 \mu \mathrm{m}, \mathrm{b}: 12 \mu \mathrm{m}$, c: $8 \mu \mathrm{m})$, under otherwise identical experimental conditions. The shorter the terrace, the less the pancake spreads, resulting in a smaller bubble. In (a), the terrace width is too large to allow a bubble to form, resulting in complete spreading.

silicon edge (dark line on Fig. 3a, corresponding to a Laplace pressure $P_{\mathrm{L}, \max }=\gamma\left(1+\cos \theta_{g}\right) / h$ with $\gamma$ being the surface tension). Any further increase in pressure would create an imbalanced meniscus, leading to gas overflowing into the foam collection channel, followed by pinch-off and the formation of a new bubble. Prior to pinch-off, the portion of the meniscus still located on the silicon terrace maintains its original shape and continues to advance. An increase in gas pressure is in this case accommodated by reducing the in-plane radius of curvature $R$. As evidenced in Figure 2, a bubble is formed as soon as $R$ reaches the value $R_{\min }$ corresponding to a Laplace pressure equal to $P_{\mathrm{L}, \max }$.

Three interesting cases appear here. If the terrace width $w_{t}$ is small enough, such that the Laplace pressure

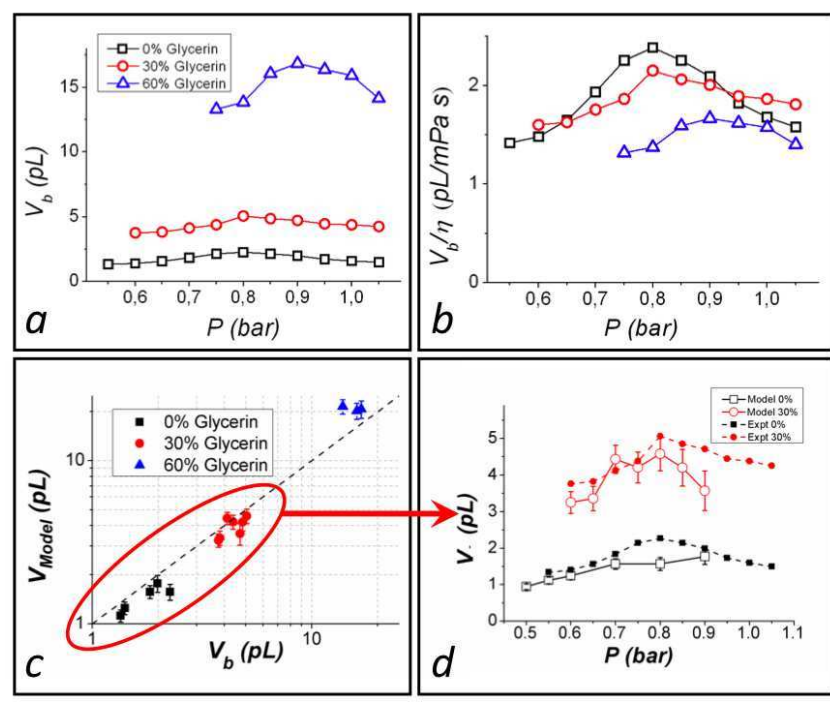

FIG. 5: Experimentally measured bubble volumes $V_{b}$ (a) for the $2.0 \mu \mathrm{m}$ device, and the volumes scaled by viscosity of the liquid $V_{b} / \eta$ (b). In (c-d) we show the predictions of the phenomenological model vs. experimental data (the model data is based on the measurements of pinned meniscus length from Fig. 3c and assumes $\alpha=60$ )

corresponding to $R_{\text {Touch }}$ is larger than $P_{\mathrm{L}, \max }$ (or, equivalently, $\left.R_{\text {Touch }}<h /\left(1-\cos \theta_{s}\right)\right)$, the bubble is formed as soon as the pancake reaches the edge; in this case, the device geometry and performance approach that of a T-junction, with the consequent sensitivity of bubble size to process parameters [2]. If, on the other hand, the terrace width is too large $\left(w_{t}>2 h /\left(1-\cos \theta_{s}\right)\right)$, purely geometrical considerations will prevent $R$ from reaching $R_{\min }$. In this case, the pancake will completely invade the terrace (Figure 4a) and polydisperse bubbles will be created in a chaotic fashion. The intermediate case is of particular interest: in this case, shown in Figure 4b-c, the terrace width (as well as other geometrical details, such as the junction between the production channel and the terrace) will dictate how far the pancake needs to spread before a bubble can be formed. The pancake will form an interface with no in-plane curvature at the terrace edge. Once the radius reached $R_{\min }$, the pressures will be imbalanced along the full length $L_{\text {pinch-off of this }}$ apparently flat interface and the bubble will overflow into the foam collection channel as a gas sheet. Experiments show that $L_{\text {pinch-off }}$ decreases with increasing gas pressure $P$ (Figure $3 \mathrm{c}$ ), which will have profound implications on the performance of the device - we presume that this effect is due to the dynamics of contact line motion.

The overflow along the pinned pancake interface results in a highly asymmetric gas sheet, with the pinchoff time $t_{\text {pinch-off }}$ being to first order proportional to $L_{\text {pinch-off }}$, the larger dimension of the sheet. Pinchoff speed is in turn determined by the ratio of surface 
tension $\gamma$ to the viscosity $\eta$. To first order, therefore, $t_{\text {pinch-off }}=\alpha L_{\text {pinch-off }} \eta / \gamma[20], \alpha$ being a dimensionless proportionality factor. The value of $\alpha \approx 60$ was obtained by estimating $t_{\text {pinch-off }}$ from analysis of high-speed camera images. The bubble volume will be related to the pinch-off time, which can be thought as the time during which gas will "blow" into the bubble; the volume of the pancake, while also contributing to the final bubble volume, is insignificant by comparison. The volumetric flowrate of gas blown into the bubble during pinch-off can be calculated by using the isothermal Hagen-Poiseuille formula corrected for gas compressibility [21]

$$
\phi_{g}(t)=\frac{d V_{b}(t)}{d t}=\frac{\left(P_{g}-P_{b}(t)\right)\left(P_{g}+P_{b}(t)\right)}{2 P_{b}(t) R_{h}}
$$

with $R_{h}$ being the hydrodynamic resistance of the gas channel connected to the bubble, $P_{g}=P_{0}+P$ being the absolute gas pressure, and $P_{b}(t)=P_{0}+$ $2 \gamma /\left(3 V_{b}(t) / 4 \pi\right)^{1 / 3}$ being the pressure inside the bubble (including the Laplace pressure, surface tension being measured for the three solutions $\gamma=35 \mathrm{mN} / \mathrm{m}$ ). The pressure drop due to fluid flow in the outlet channel is several orders of magnitude lower and will be ignored in the following. Figure 5a displays the measured bubble volume for the three solutions used in this study, a range of gas pressures, and the liquid flowrate fixed at $300 \mu \mathrm{l} / \mathrm{s}$; in all cases, under fixed process parameters, the bubbles created were very monodisperse (below the experimental detection limit imposed by the image pixel size, $C V<1 \%$; several hundreds of images obtained under identical process conditions were used for this measurement). Regarding the dependence on process parameters, we notice that the bubble volume does not increase uniformly with gas pressure as might be expected, but displays a maximum value at gauge pressures $P \approx 0.8-0.9$ bar. As Figure $5 \mathrm{~b}$ shows, bubble volumes roughly scale with viscosity, which confirms our presumption that most of the gas enters the bubble during the viscosity-controlled pinch-off time (in a first-order approximation, ignoring Laplace pressure, bubble volume is proportional to $\left.t_{\text {pinch-off }}\right)$. Finally, Figure $5 \mathrm{c}$ shows the comparison between the phenomenological model outlined above and experimental data, $V_{\text {Model }}$ being obtained using numerical integration of Eq.1 from $t=0$ to $t_{\text {pinch-off }}$, for $\alpha=60$. Figure $5 \mathrm{~d}$ provides a detailed comparison of the model and experimental data, for the lower two pressures. Empty circles show $V_{\text {Model }}$, whereas filled circles show the experimental data. We notice good agreement, and particularly, the prediction of a maximum bubble volume consistent with experimental data. The explanation of this behavior stems from the fact that while $P$ increases, $L_{\text {pinch-off }}$ decreases (Figure $3 c$ ), bubble volume depending roughly on their product. This simple model ignores a number of elements such as the effects of specific geometry details, of dynamic contact angle during "pancake spreading", and of the dynamic surface tension during the rapid pinch-off; these will be incorporated in further model refinements.

Production of small monodisperse bubbles using microchannel emulsification geometries is possible, but the production mechanism is relatively complex. A simple phenomenological model relying on the existence of a pinned gas-liquid interface seems to correctly predict the qualitative behavior of the bubble generator. The detailed geometry of the device is critical in determining device performance. If the production throughput of such devices could be scaled up while maintaining the observed $<1 \%$ monodispersity, they might find uses in generating photonic crystals, or for therapeutical applications such as drug vectors, artificial blood, or ultrasonic contrast agents. The scaling up of production in parallelized geometries is not a trivial issue due to the different coupling mechanisms between individual generators. It is possible to effectively decouple the production at individual sites, but this development requires complex devices with non-planar topologies. Our work in this direction will be presented in a separate manuscript.

We acknowledge support from Institut Carnot-Vitres and we thank the reviewers for very constructive comments.

[1] T. Nisisako, T. Torii, T. Higuchi, Lab Chip 2, 24 (2002).

[2] P. Garstecki, M.J. Fuerstman, H.A. Stone, G.M. Whitesides, Lab Chip 6, 437 (2006).

[3] S.L. Anna, N. Bontoux, H.A. Stone, Appl. Phys. Lett. 82, 364 (2003).

[4] P. Garstecki et al., Appl. Phys. Lett. 85, 2649 (2004).

[5] E. Lorenceau et al., Phys. Fluid. 18, 097103 (2006).

[6] E. Castro-Hernandez, W. van Hoeve, D. Lohseb and J.M. Gordillo, Lab Chip 11, 2023 (2011)

[7] M. Seo et al., Langmuir 26, 13855 (2010)

[8] R.M. Lorenz et al., Anal. Chim. Acta 630124 (2008).

[9] T. Nisisako and T. Torii, Lab Chip 8, 287 (2008).

[10] V. Barbier, H. Willaime, P. Tabeling, F. Jousse, Phys. Rev. E 74, 046306 (2006).

[11] M. Hashimoto et al., Small 4, 1795 (2008).

[12] M.T. Sullivan, H.A. Stone, Phil. Trans. R. Soc. A 366, 2131 (2008).

[13] I. Kobayashi, S. Mukataka, M. Nakajima, Langmuir 21, 7629 (2005);

[14] S. Sugiura et al., Langmuir 17, 5562 (2001).

[15] I. Kobayashi, Y. Wada, K. Uemura and M. Nakajima, Microuid Nanouid 8, 255 (2010).

[16] I. Kobayashi, S. Mukataka, M. Nakajima, Ind. Eng. Chem. Res. 44, 5852 (2005).

[17] K. van Dijke, K. Schroen and R. Boom, Langmuir, 24, 10107 (2008).

[18] E. van der Zwan, K. Schroen and R. Boom, Langmuir, 25, 7320 (2009).

[19] K. van Dijke et al., Microfluid Nanofluid, 9, 77 (2010).

[20] J. Eggers, Rev. Mod. Phys., 69, 865 (1997).

[21] R.K. Prud'homme, T.W. Chapman and J.R. Bowen, Appl. Sci. Res., 43, 67 (1986) 